\title{
The general practitioners' view
}

\author{
Trevor Cull \\ M.B., Ch.B. \\ General Practitioner, \\ 114 Middleton Hall Road, Birmingham, 30
}

I AM conscious of the recognition you have bestowed upon the general practitioners in asking me to this Conference, for it implies, I believe, a recognition that we are not only consumers of 'casualty services' but can make a real contribution to the solution of some of the problems.

My remit is to tell you what we, as family doctors, want from the casualty departments and to discuss amongst other things the feasibility of these departments being run by general practitioners.

May I first state the current problem as I see it.

There is, I believe, little doubt that the patient has become hospital orientated. It would appear that the old image of the general practitioner as portrayed by Doctor Cameron has faded in recent years, perhaps under the barrage of the popular press and television and given way to a new image - that of the handsome young Doctor Kildare working in an attractive, glamorous, antiseptic and above all, successful atmosphere of a modern hospital.

It is natural therefore that the patients, not finding these facilities available at their general practitioners' surgeries should turn to the public hospitals for advice.

In a small series of 249 medical cases coming to Stoke Hospital in $1968,30 \%$ only were seen and referred by the general practitioners. Of the other $70 \%$, some $10 \%$ had tried to get their doctor and found him not available but the large majority of this group, that is $90 \%$, had not even attempted to contact the family doctor at all. This high percentage of self referral to the casualty departments is I believe due to two factors:

(a) The lack of availability of the general practitioners-and it is important to remember that a large part of the general practitioner's time is taken up with home visits; and

(b) The glamour factor of the hospital previously referred to.

It is not surprising therefore that the family doctor is blamed by the staff in the casualty departments for using the hospital as cheap cover for his surgeries. It is pertinent to ask, what does the reasonable general practitioner want from the casualty department? The list could of course be endless, but I think there are three major services which the general practitioner may reasonably expect of his local department:

(a) He must be able to refer minor trauma to the department for radiological examination. I think even at this Conference there is little need for me to remind you of the 'therapeutic' value of an X-ray, but it is also important to remember that the general practitioner is equally as vulnerable as the casualty doctor to litigation.

(b) Minor infections requiring incision and drainage under general anaesthetic must be referable to the casualty department for treatment.

(c) He must be able to refer a case for an urgent second opinion: the doubtful abdomen being an example of this type of patient.

These are my own opinions and in order to clarify those of my colleagues, in October 1971 I questioned a group of fifty-three general practitioners at the Postgraduate Centre, Selly Oak, Birmingham. Replying to the question 'What does the family doctor want from casualty?' the following answers were given:

(1) X-ray.

(2) Reception of trauma of all kinds.

(3) Facilities for stomach wash-outs.

(4) Suturing of lacerations.

(5) Excision of cysts.

(6) Incision of septic cysts.

(7) Treatment of epistaxis.

(8) Treatment of foreign bodies in the eye.

(9) Ability to refer patients for physiotherapy.

(10) Provision of sterile packs.

It is often stated that the general practitioner must come back into the hospital, and some believe that his place within the hospital is in the casualty department. I can of course speak only for my immediate colleagues in the south-west suburban ward of Birmingham who number some 102 doctors. Twentyseven of these doctors hold clinical assistant appointments at local hospitals, of which only four are in the casualty department. However, we do run our own coronary care ambulance staffed by twenty-two general practitioners on a rota, but when being asked if they would consider being responsible for the casualties on a similar 24-hr basis, the answer was a 
most definite 'NO'. So, any 'solution' based on the voluntary staffing of the casualty departments by general practitioners, is, I think, a non-starter. I think one could raise two or three doctors who would do short periods of 'on call', that is all.

How can the general practitioners help?

I believe that if the health centre concept of family doctoring was implemented, this would relieve the local casualty department of a considerable burden. Such health centres would give at least a 12-hr service and being adequately staffed, would increasingly undertake minor surgery. However, it is important to realize that if a health centre is going to be staffed by a group of eight or more general practitioners then the area this health centre serves may be considerable and certainly well beyond the 'pram pushing' range of young mothers. Transport to such centres must become available in order to reduce the excessive burden of home visits, simply because it is inconvenient for the patient to attend surgery.

Secondly, we must have open access to radiological facilities, if not for contrast media examina- tions then at least for simple X-rays for bone injuries and some reasonably quick means of getting a report on the X-rays within $48 \mathrm{hr}$.

If these two measures were implemented we should eliminate $75 \%$ of the 'casuals' presenting at casualty departments in the large cities. For most of these patients, knowing the limitations of the surgeries both in time and facilities, present themselves to the casualty departments.

Finally, I have been asked to consider the question of rural casualties.

My contacts within rural areas are few, but my impression is that the family doctor does in fact provide 24-hr cover for his own patients which may extend to his colleagues if they are not available. However, talking to doctors in the area of Evesham, Stratford, Rhayader, I have the inescapable feeling that the patients in such places are far less demanding on their doctors and that, should they present to the doctor or the local hospital outside of normal consultation hours, then they really are a little 'poorly' and have something more than herpes zoster. 